\title{
An optical fiber Bragg grating tactile sensor
}

\author{
Barbara Cowie $^{a}$, Thomas Allsop ${ }^{a}$, John Williams ${ }^{a}$, David $\mathrm{Webb}^{a}$, \\ Ian Bennion ${ }^{a}$ and Matthew Fisher ${ }^{b}$ \\ ${ }^{a}$ Aston University, Birmingham, B4 7ET. UK \\ ${ }^{b}$ Aerovac Systems Ltd, Keighley, BD20 5NG. UK
}

\begin{abstract}
Tactile sensors are needed for many emerging robotic and telepresence applications such as keyhole surgery and robot operation in unstructured environments.

We have proposed and demonstrated a tactile sensor consisting of a fibre Bragg grating embedded in a polymer "finger". When the sensor is placed in contact with a surface and translated tangentially across it measurements on the changes in the reflectivity spectrum of the grating provide a measurement of the spatial distribution of forces perpendicular to the surface and thus, through the elasticity of the polymer material, to the surface roughness.

Using a sensor fabricated from a Poly Siloxane polymer (Methyl Vinyl Silicone rubber) spherical cap $50 \mathrm{~mm}$ in diameter, $6 \mathrm{~mm}$ deep with an embedded $10 \mathrm{~mm}$ long Bragg grating we have characterised the first and second moment of the grating spectral response when scanned across triangular and semicircular periodic structures both with a modulation depth of $1 \mathrm{~mm}$ and a period of $2 \mathrm{~mm}$. The results clearly distinguish the periodicity of the surface structure and the differences between the two different surface profiles. For the triangular structure a central wavelength modulation of $4 \mathrm{pm}$ is observed and includes a fourth harmonic component, the spectral width is modulated by $25 \mathrm{pm}$.

Although crude in comparison to human senses these results clearly shown the potential of such a sensor for tactile imaging and we expect that with further development in optimising both the grating and polymer "finger" properties a much increased sensitivity and spatial resolution is achievable.
\end{abstract}

Keywords: Fibre Bragg Grating sensor, tactile sensor, optical fibre sensor

\section{INTRODUCTION}

Tactile sensors are needed for many emerging robotic and telepresence applications such as keyhole surgery and robot operation in unstructured environments. The artificial sensing of surface texture (or surface profile) is also of great interest to industries such as road surfacing, ${ }^{1,2}$ textiles and abrasives, and in the area of machined finishes. Fine surface finish information for machined surfaces (on the scale of $0.2-5 \mu \mathrm{m})^{3}$ is commonly measured using optical methods such as interferometry. Larger surface details $(0.1-5 \mathrm{~mm})$ are not easily characterisable in this

Author contact: cowiebm@aston.ac.uk 

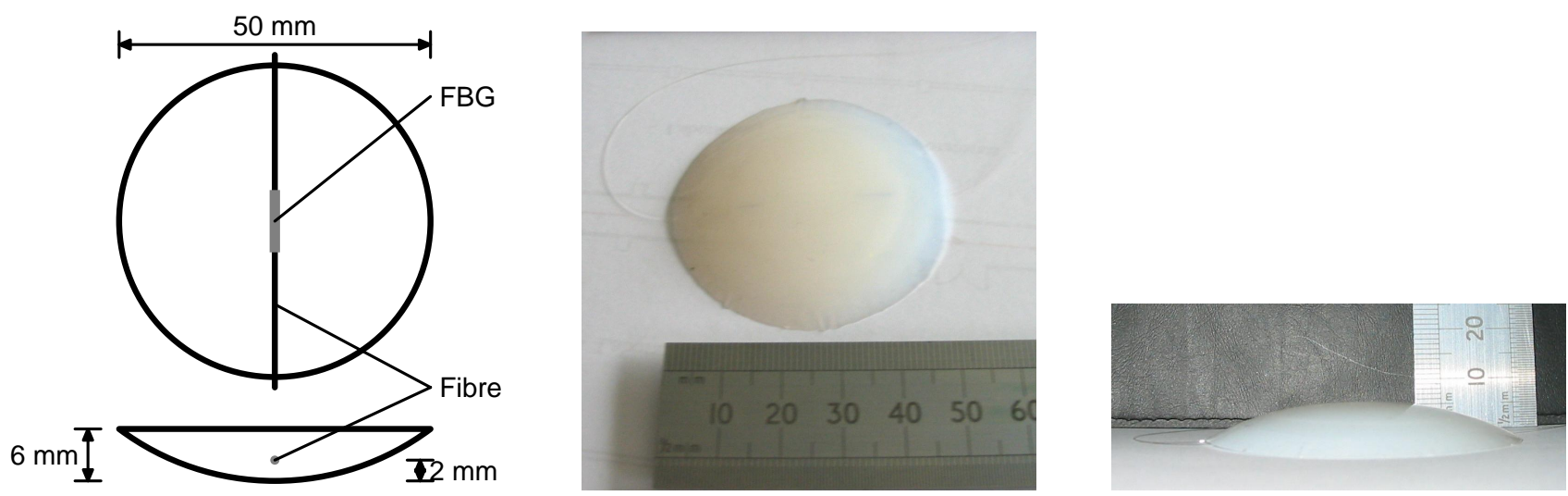

Figure 1. Poly Siloxane polymer sensor encapsulation

way. The human finger, which is a very good sensor of surface texture on this scale utilises at least 4 kinds of mechanoreceptors ${ }^{4}$ densely distributed across the fingertip. Attempts to emulate the human system have used polyvinylidene fluoride films ${ }^{5,6}$ vertical sampling pressure probes, ${ }^{7}$ strain gauges ${ }^{8}$ and a piezoelectric microphone. ${ }^{9}$ Many tactile sensors capable of detecting small scale profile details consist of arrays of many sensors which present problems in terms of connection for information retrieval and require computationally intensive signal processing.

The sensor we propose has one embedded optical fibre Bragg grating (FBG) sensing element with a single optical fibre connection. In addition to the advantages of reduced connectivity requirements, it requires no electrical power at the sensor point and is immune to electromagnetic interference which would be advantageous in many environments.

\section{SENSOR CONSTRUCTION AND CHARACTERISATION}

The sensors consisted of fiber Bragg gratings embedded in Poly Siloxane polymer (Methyl Vinyl Silicone rubber) spherical caps typically $50 \mathrm{~mm}$ in diameter and $6 \mathrm{~mm}$ thick as shown in figure 1 . The FBGs were fabricated in standard single mode optical fibre, previously hydrogenated, using a $244 \mathrm{~nm}$ laser and a phase mask. After fabrication they were annealed at $80^{\circ} \mathrm{C}$ for 24 hours before encapsulation in the polymer cap. A range of sensors with varying grating lengths were fabricated and tested.

When the polymer cap "finger" is pressed into contact with the surface to be measured it's elastic properties convert deformation due to protruding surface features into internal strain resulting in variation in the distributed strain along the FBG and hence, into changes in its reflected spectrum.

The reflected optical power spectra from the FBGs were measured using a broadband light source (AFC Technologies BBS 1550) and an optical spectrum analyser (OSA) (HP 70004A). The spectra were characterised by their central wavelength (the first moment of the power spectrum) and their rms spectral width. 


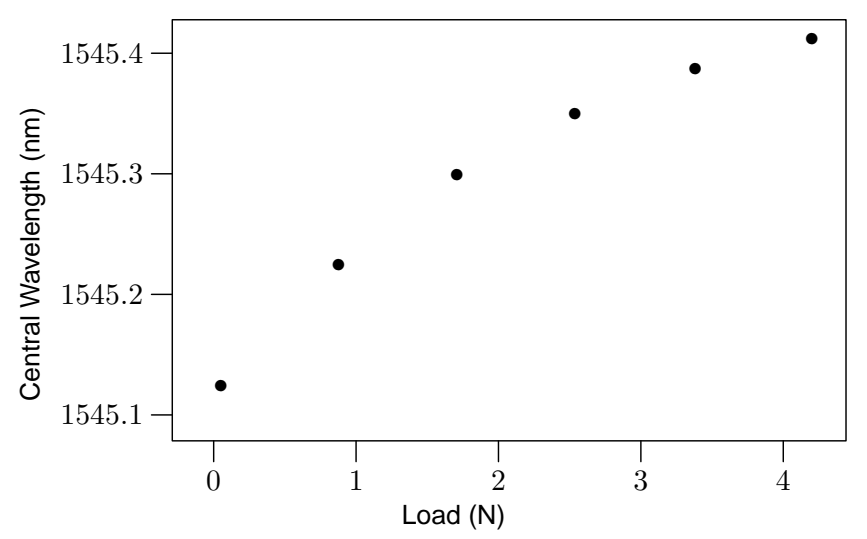

Figure 2. Variation of central wavelength versus applied load for a $10 \mathrm{~mm} \mathrm{FBG} \mathrm{sensor}$

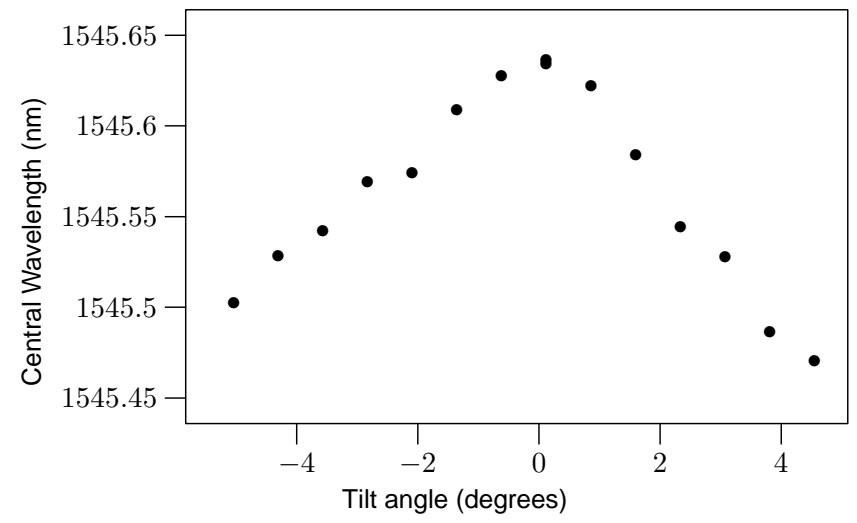

Figure 3. Variation of central wavelength versus sensor tilt relative to applied force for a $10 \mathrm{~mm}$ FBG sensor

Figures 2 and 3 provide characterisation of a sensor using a $10 \mathrm{~mm}$ FBG encapsulated grating under static load conditions.

Figure 2 shows how the central wavelength of the grating spectrum varied with load applied at the apex of the sensor cap and perpendicular to it. This clearly shows that the response of the grating is not linear with applied force. Since the response of an FBG to strain is linear (in the elastic limit) at constant temperature ${ }^{10}$ this must be due to nonlinearity in the compression of the silicone rubber due to the device geometry.

Figure 3 shows how the central wavelength varies under a constant load of $3.37 \mathrm{~N}$ as the direction of the applied load was varied away from the perpendicular.

Similar plots are obtained for grating bandwidth. These results clearly illustrated the sensitivity of the device to the magnitude and direction of the applied load. 


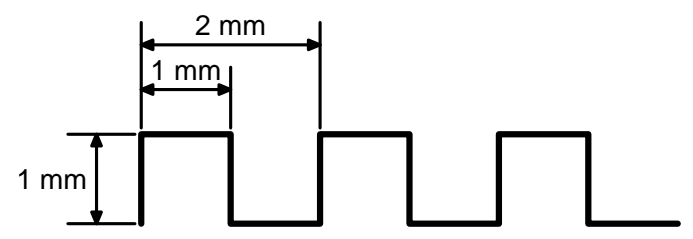

(a) Square

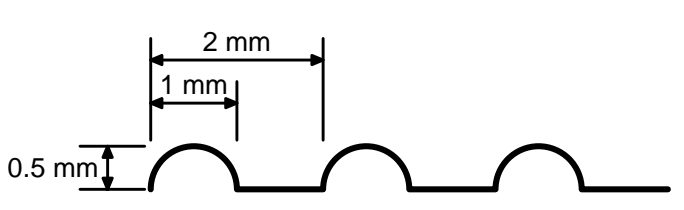

(c) Semicircular

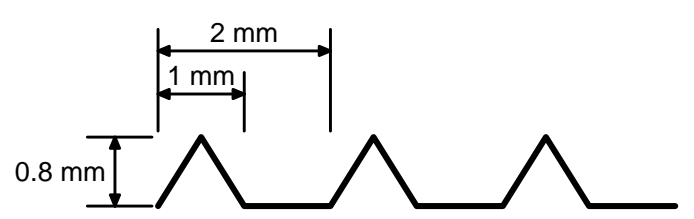

(b) Triangular

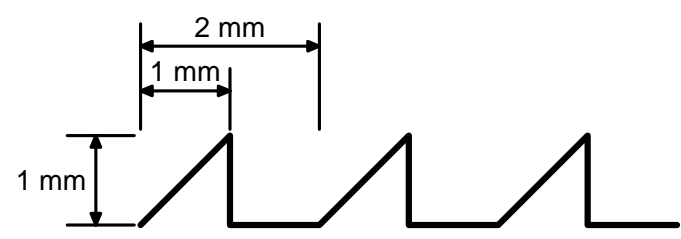

(d) Sawtooth

Figure 4. Surface Profiles

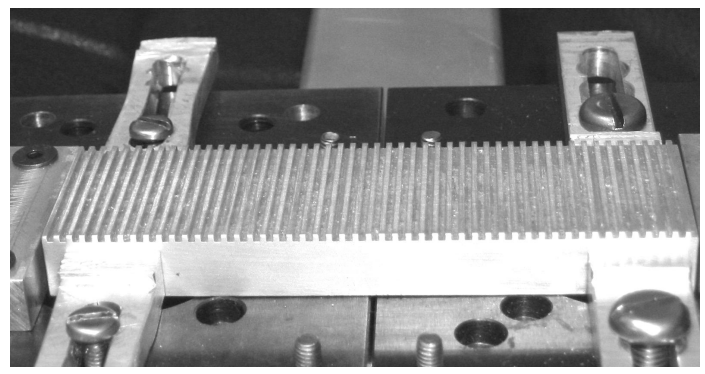

Figure 5. Machined block with the periodic square surface profile

\section{MEASUREMENT OF SURFACE PROFILES}

If the sensor is impressed onto a rough surface and then translated tangentially across it the distribution and magnitude of the forces applied through the polymer cap in contact with the surface and through the elasticity of the polymer will change the distributed strain and thus reflectivity spectrum of the FBG. Thus the characterisation of how the spectrum varies as the sensor is translated provides a measurement of the roughness of the surface.

In order to test the effectiveness of the sensor, a range of surfaces were machined in aluminium blocks to provide a repeating pattern of ridges of varying profile. Figure 4 gives the dimensions of the profiles used in the work reported here. Figure 5 shows, as an example, a photograph of the square profiled block.

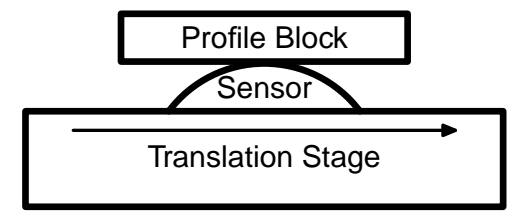

Figure 6. Translation of the sensor across the profiled surface 


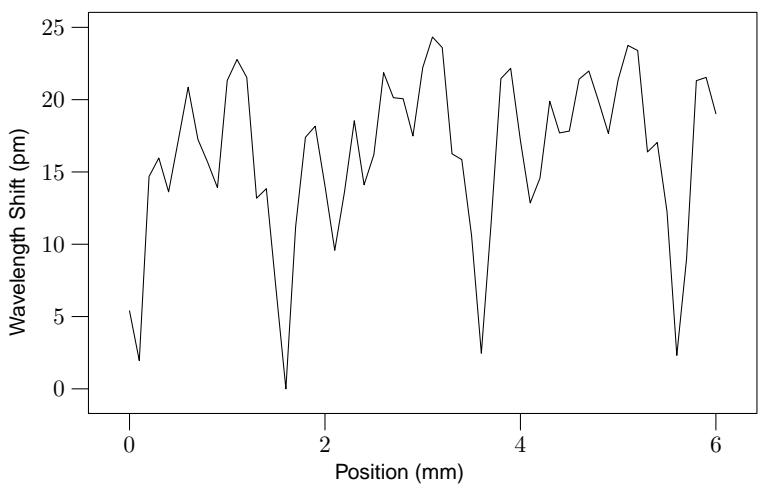

(a) Shift in Central Wavelength

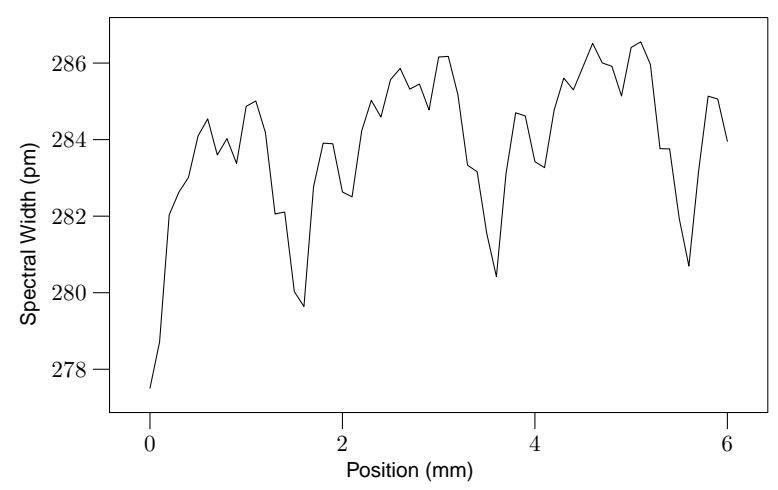

(b) Spectral Width

Figure 7. Spectral characteristics versus displacement for the triangular profile surface, $10 \mathrm{~mm}$ sensor

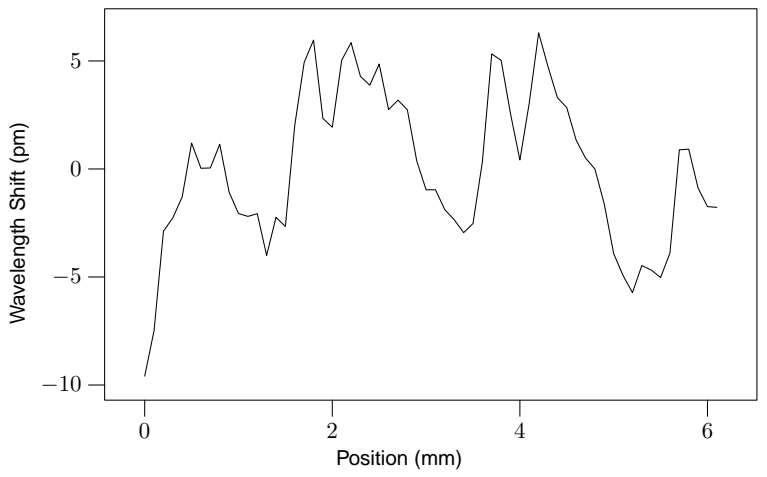

(a) Shift in Central Wavelength

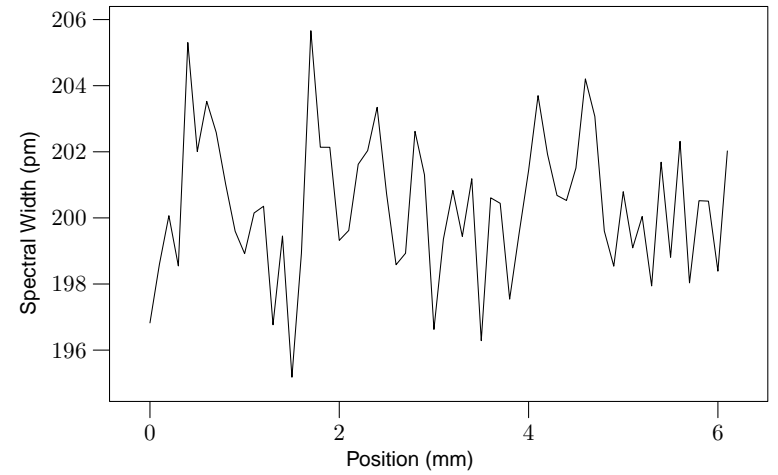

(b) Spectral Width

Figure 8. Spectral characteristics versus displacement for the semicircular profile surface (with linear trend removed), $10 \mathrm{~mm}$ sensor

The sensor was clamped to a translation stage with the profile blocks mounted above the sensor such that the sensor made contact with the block but was still able to move freely beneath it as illustrated in figure 6. The force was adjusted so that the pressure of the block caused the spectral peak reflected by the grating to shift by $300 \mathrm{pm}$. The profile block was adjusted to be parallel to direction of motion of the translation stage, and the grating reflectivity spectra recorded at intervals over a $6 \mathrm{~mm}$ (3 period) range of translation. The fiber grating and direction of motion were parallel to each other and perpendicular to the direction of the grooves in the profile blocks.

The initial sensor had a $10 \mathrm{~mm}$ long embedded Bragg grating. The resulting shift in the spectral centre and width versus position are given in figures 7,8 and ,9 for the triangular, semicircular and sawtooth profiled surfaces respectively. In figure 7 we see a slight linear trend across the spectral characteristics arising due to the direction of translation not being perfectly parallel to the surface. This trend was removed from subsequent results to leave only the features corresponding to the surface profile characteristics. The square profile gave no perceptible periodic response and is not shown, possibly because the recesses in the profile were smaller 


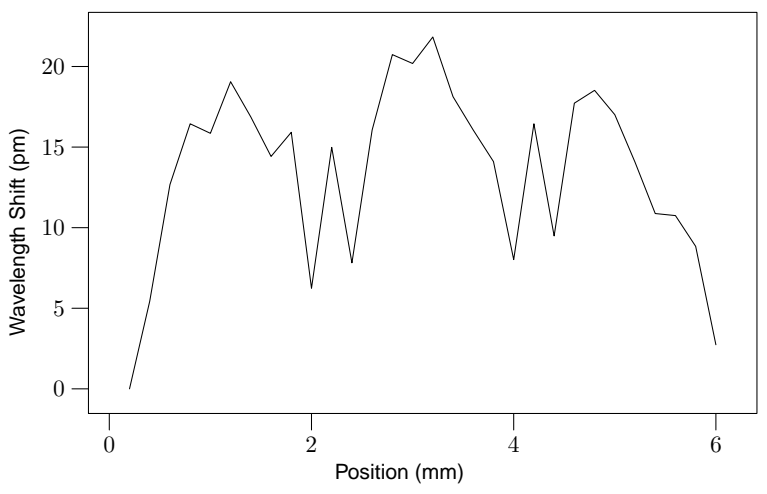

(a) Shift in Central Wavelength

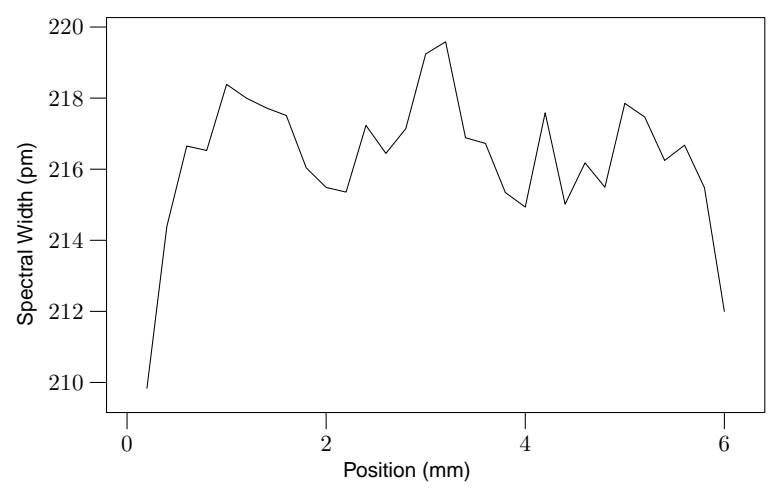

(b) Spectral Width

Figure 9. Spectral characteristics versus displacement for the sawtooth profile surface (with linear trend removed), $10 \mathrm{~mm}$ sensor

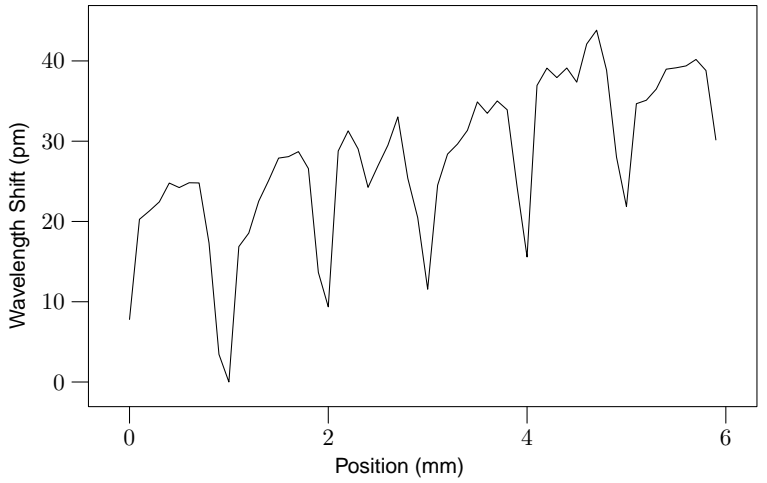

(a) Shift in Central Wavelength

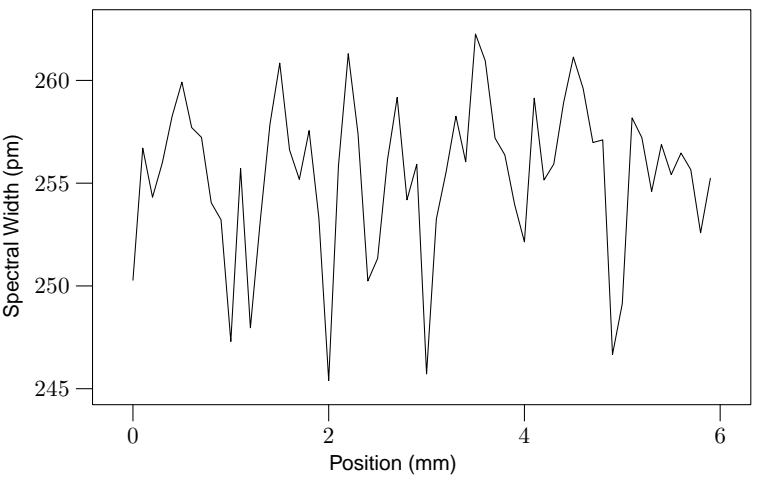

(b) Spectral Width

Figure 10. Spectral characteristics versus displacement for the triangular profile surface, $25 \mathrm{~mm}$ sensor

( $1 \mathrm{~mm}$ wide) than the recesses in the other profiles, so that the silicone was less able to deform into them.

For each surface profile a clear periodicity corresponding to the periodicity in the surface profile is seen in both the wavelength shift and spectral width, and the periodic spectral changes measured versus displacement are clearly different in shape and magnitude for the different surface profiles. Thus, from these spectral measurements, we can determine both the feature size and some information about the shape of the surface features.

We also see harmonic components in the measured spectral changes, which implies that we should be able to measure surface profiles with higher spatial frequencies, smaller features than we have demonstrated using the machined blocks here.

We also performed measurements using sensors which had $25 \mathrm{~mm}$ FBG embedded in the polymer caps. Figures 10 and 11 show the measurements obtained with these devices. A longer fiber Bragg grating has a narrower bandwidth and thus can be used to measure more sensitivelychanges in the spectrum and thus smaller changes in strain. However, being of larger 


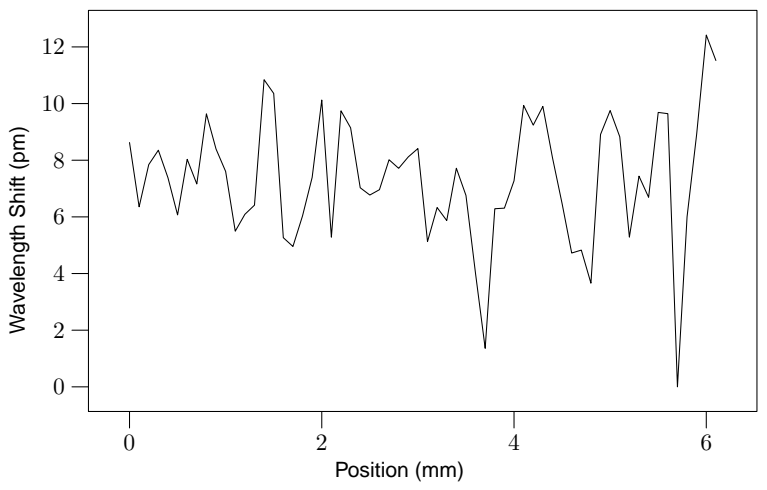

(a) Shift in Central Wavelength

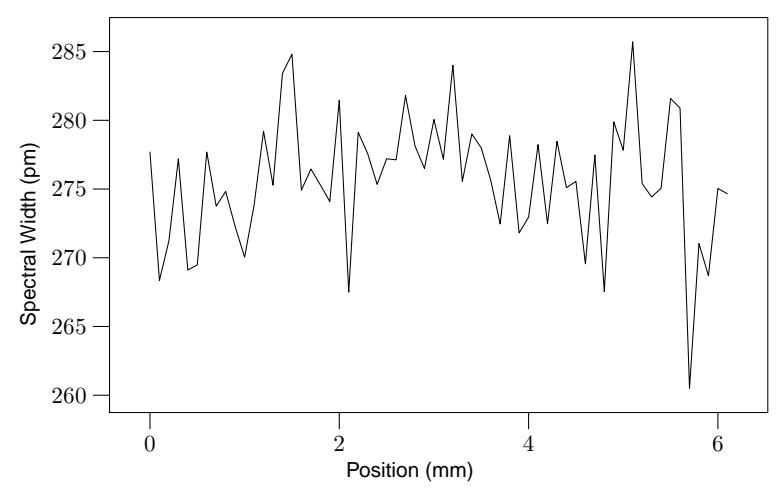

(b) Spectral Width

Figure 11. Spectral characteristics versus displacement for the semicircular profile surface, $25 \mathrm{~mm}$ sensor (linear trend removed)

extent may mean that we lose spatial resolution as we are measuring the spectral characteristics integrated across the whole grating length. For the $25 \mathrm{~mm}$ sensor the grating length is $12.5 \times$ longer than the surface profile period being measured yet we clearly see a peridocity in the spectral characteristics. The magnitude of the measured spectral shift is larger for the the $25 \mathrm{~mm}$ FBG than for the shorter $10 \mathrm{~mm}$ FBG sensor. The optimum design of these sensors is therefore not intuitive and must take account of the physical characteristics of the polymer cap as well as the response of an FBG to a complex distributed strain.

\section{CONCLUSIONS}

The tactile sensor described above has been found to be responsive to applied load, the angle of an applied load, and to have the potential to distinguish between different surface profiles. Further work is necessary to optimise the length of the grating, the shape of the silicone pad, the hardness of the silicone material and various other factors, however it has been demonstrated that this approach to tactile sensing shows promise, particularly for use in medical applications such as minimally invasive surgery, were the single connection required to this sensor would be very attractive in endoscopy, where space is at a premium, and in applications such as MRI scanners, where the immunity to electromagnetic interference would be an asset. It may also be useful in industrial applications such as textiles manufacture and surface finish evaluation.

\section{ACKNOWLEDGMENTS}

Barbara Cowie is supported by the EPSRC.

\section{REFERENCES}

1. D. I. Hanson and B. D. Prowell, "Evaluation of circular texture meter for measuring surface texture of pavements," tech. rep., National Center for Asphalt Technology, Auburn University, Auburn, Alabama, September 2004. NCAT Report 04-05. 
2. "Strategic plan for improved concrete pavement surface characteristics." http://www.cptechcenter.org/publications/ surface_char_exec_summary.pdf, July 2006.

3. P. Tomassini, L. Rovati, G. Sansoni, and F. Docchio, "Novel optical sensor for the measurement of surface texture," Review of Scientific Instruments 72, pp. 2207-2213, April 2001.

4. B. V. Jayawant, "Tactile sensing in robotics," Journal of Physics. E: Scientific. Instruments 22(9), pp. 684-692, 1989.

5. J. Dargahi and S. Payandeh, "Surface texture measurement by combining signals from two sensing elements of a piezoelectric tactile sensor.," in Proceedings of the SPIE, 3376, pp. 122-128, The International Society for Optical Engineering, 1998.

6. Y. Tada, K. Hosoda, Y. Yamasaki, and M. Asada, "Sensing the texture of surfaces by anthropomorphic soft fingertips with multi-modal sensors," in Proceedings 2003 IEEE/RSJ International Conference on Intelligent Robots and System, 1, pp. 27-31, (Las Vegas, USA), October 2003.

7. H. Shinoda, M. Uehara, and S. Ando, "A tactile sensor using three-dimensional structure," in Proceedings, 1993 IEEE International Conference on Robotics and Automation, 1, pp. 435-441, IEEE, May 1993.

8. Y. Mukaibo, H. Shirado, M. Konyo, and T. Maeno, "Development of a texture sensor emulating the tissue structure and perceptual mechanism of human fingers," in Proceedings of the 2005 IEEE International Conference on Robotics and Automation, pp. 2576-8251, IEEE, (Barcelona, Spain), April 2005.

9. D. K. Pai and P. R. Rizun, "The what: A wireless haptic texture sensor," in Proc. Eleventh Symposium on Haptic Interfaces for Virtual Environment and Teleoperator Systems, 2003.

10. X. Zhang, Z. Wu, and B. Zhang, "Strain dependence of fiber bragg grating sensors at low temperature," Optical Engineering 45, may 2006. 